\title{
Brain Tumor Analysis of Rician Noise Affected MRI Images
}

\author{
Renukalatha S. \\ Associate Professor, Dept. of CS \& E, \\ Sri Siddhartha Institute of Technology, \\ Tumkur, India
}

\author{
K.V. Suresh \\ Senior Member IEEE \& Professor, \\ Dept. of EC \& E, \\ Siddaganga Institute of Technology, \\ Tumkur, India
}

\begin{abstract}
Magnetic Resonance Imaging (MRI) established itself as a key imaging modality in diagnosis and treatment of brain tumors. Automatic segmentation of tumors becomes a tedious task due to complex anatomical brain structure. In addition, presence of noise degrades the quality of MRI scans. MRI images are usually corrupted by Rician noise which would mislead the image analysis algorithms and results in improper diagnosis of the diseases. Also, poor tumor boundary becomes a major hurdle for the subsequent stages of tumor analysis such as: feature extraction, classification and quantification. Classification accuracy mainly depends on quality of the denoised images and sharpness of the tumor boundary. This paper investigates the performance evaluation of different image matting techniques to extract tumor from Rician noise affected MRI brain images.
\end{abstract}

\section{General Terms}

Medical Image Processing

\section{Keywords}

MRI Brain tumor, Rician noise, Region of interest (ROI), image matting and Sensitivity.

\section{INTRODUCTION}

Computer-aided diagnosis (CAD) is usually used as a second opinion by medical experts in brain tumor detection and quantification [1]. Brain tumor is a group of defective cells that grow inside the brain or around the brain. Tumors can be benign (noncancerous) or malignant (cancerous). In the last decade, MRI established itself as a key imaging modality in diagnosis and treatment of brain tumors. Due to high structural complexity, these images suffer from intensity inhomogeneity and partial volume effect. Further, presence of noise degrades the quality of these images. Extracting tumor regions from such noisy images retaining the signal features is highly challenging issue. Poor tumor boundary is an obstacle for effective feature extraction, analysis, recognition and quantitative measurements. Accuracy and time complexity of analysis stage mainly depends on the noise removal techniques and accuracy of the boundary of ROI.

In brain MRI analysis, image segmentation is commonly used for: measuring and visualizing the brain's anatomical structures, analyzing the growth of the tumor, proper treatment planning and image-guided surgeries. Since last two decades, various segmentation techniques of different accuracy and degree of complexity have been developed and reported in the literature.

Most of these techniques initially conduct the standard preprocessing steps which include image resizing, image de noising, contrast enhancement and morphological operations like dilation and erosion to remove non tumor regions, [2].
Many image processing techniques have been developed for extracting tumor ROI. To list a few, automatic segmentation of heterogeneous MRI data was proposed by Jason et al, [3] which uses Bayesian formulation incorporating soft model assignments into the calculation of pixel affinities. Tao Wang et al. [4] developed a fluid vector flow active contour model (FVFACM), which addressed the problems of traditional ACM methods having insufficient capture range and poor convergence. FVF demonstrates improvements over techniques like gradient vector flow, boundary vector flow, and magneto static active contour. Tandoori et al. [5] proposed a novel automatic segmentation approach to identify brain structures in MRIs for volumetric measurements. This method is based on active contour model combined with Support Vector Machine classifiers (SVM) and validation is done using the gold standard brain MRI data set. Jafari, et al. [6] proposed a hybrid technique employing seeded region growing segmentation (SRGS) algorithm with connected component labelling (CCL). An automated computer aided brain tumor detection using a fast boundary box algorithm was proposed by BaidyaNath et al. [7]. A multi-stage segmentation method based on super pixel and fuzzy clustering (MSFCM) [8], achieves good brain MRI segmentation results. MSFCM utilizes the super pixels as the clustering objects instead of pixels, and increases the clustering granularity to overcome the influence of noise and bias effectively. A novel approach to segment brain tumor based on local independent projection-base classification was proposed in [9]. But most of the segmentation techniques have not addressed the tumor extraction in presence of Rician noise. However, to improve the segmentation accuracy, it is most essential to reduce these noise levels initially and to segment the objects of interest at the later stages.

Noise in MR images obeys a Rician distribution [10]. The main sources of noise are, RF field generated from the electronics circuit and patient. The main objective of image denoising techniques is to remove such noise while retaining the important signal features. In particular, edges are important features for MR images and thus the denoising must be balanced with edge preservation. Many denoising filters for Rician noise removal have been reported in the literature. Nowak [11] addresses Rician noise removal in MRI images using a novel wavelet domain filter that adapts to variations of both signal and noise and found the reduction in Rician noise contamination in low and high signal conditions. Nobi et al. [12] demonstrated the Rician noise removal in MRI images combining mean and median filters. These filters are simple to understand and preserves brightness differences resulting in minimal blurring of regional boundaries. It also preserves the positions of boundaries in an image, making this method useful for visual examination and measurement. The quality of the output images is measured by the statistical measures: peak signal-to-noise ratio (PSNR) and root mean square error 
(RMSE).The main drawback of median filter is that the noisy pixels are replaced by some median value in their vicinity without taking into account local features such as presence of edges, [13]. Hence details and edges are not recovered satisfactorily, especially when the noise level is high. Strode et al. [14], proposed Non local Means (NLM) denoising method to reduce Rician noise in MRI data and the main drawback was high computational time Complexity of the algorithm.

In this paper, a combination of Gauss filter and anisotropic diffusion filter is used to reduce Rician noise in MRI brain tumor images.

The paper is organized as follows: Section 1 gives the introduction and in section 2, mathematical background of Rician noise is elaborated. Section 3 presents the methodology of the proposed system. Section 4 describes different ROI and image matting techniques used for tumor extraction with boundary refinement. Experimental results are provided in section 5 , followed by conclusion in section 6 .

\section{MATHEMATICAL FOUNDATION}

In this section, analysis of Rician noise in MRI images is revealed based on its probability density function (PDF) which is given in equation (1).

\subsection{Rician noise}

MR images are corrupted by Rician noise, which arises from complex Gaussian noise in the original frequency domain measurements [15].

$$
\begin{gathered}
p(q \mid t, \sigma)=\frac{t}{\sigma^{2}} \exp \left(-\frac{t^{2}+q^{2}}{2 \sigma^{2}}\right) I_{0}\left(\frac{t \times q}{\sigma^{2}}\right) \\
q=\sqrt{\left(t+n_{r}\right)^{2}+n^{2}}{ }_{i}
\end{gathered}
$$

where, $t$ is the true signal (pixel) intensity, $q$ is the observed image pixel intensity, $\sigma$ is the standard deviation of the Gaussian noise in the real and the imaginary images, and $I_{o}$ is the zero-order modified Bessel function of the first kind. Equation (2) represents the magnitude image equation for MRI images. A special case of the Rician distribution is in image regions where only noise is present and $S N R=$ $q / \sigma=0$ (e.g., in the dark background areas of an MRI where no signal is present).This special case of the Rician distribution where $q=0$ and $I_{o}=1$ is also known as the Rayleigh distribution:

$$
f_{\text {Rayleigh }}(s)=\frac{s}{\sigma^{2}} \exp -\frac{s^{2}}{2 \sigma^{2}}
$$

In the image regions where the signal is present and $\mathrm{SNR} \geq 3$, the noise distribution approximates a Gaussian distribution. Thus, the problem of Rician noise in the brain MRI is often simplified in practice by assuming the Gaussian distribution for the noise, [16].

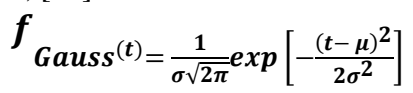

\section{METHODOLOGY}

The flow diagram of the proposed methodology is as shown in Fig 1. Initially, noisy brain tumor images are preprocessed using Gauss filter and anisotropic diffusion filter to remove Rician noise. Further, contrast of the denoised image is enhanced and the tumor ROI is detected using Otsu's thresholding and region growing algorithms. Later, the boundary information of the tumor ROI is marginally improved using three different image matting techniques. Finally, Sensitivity of segmented tumor is evaluated before and after image matting techniques.

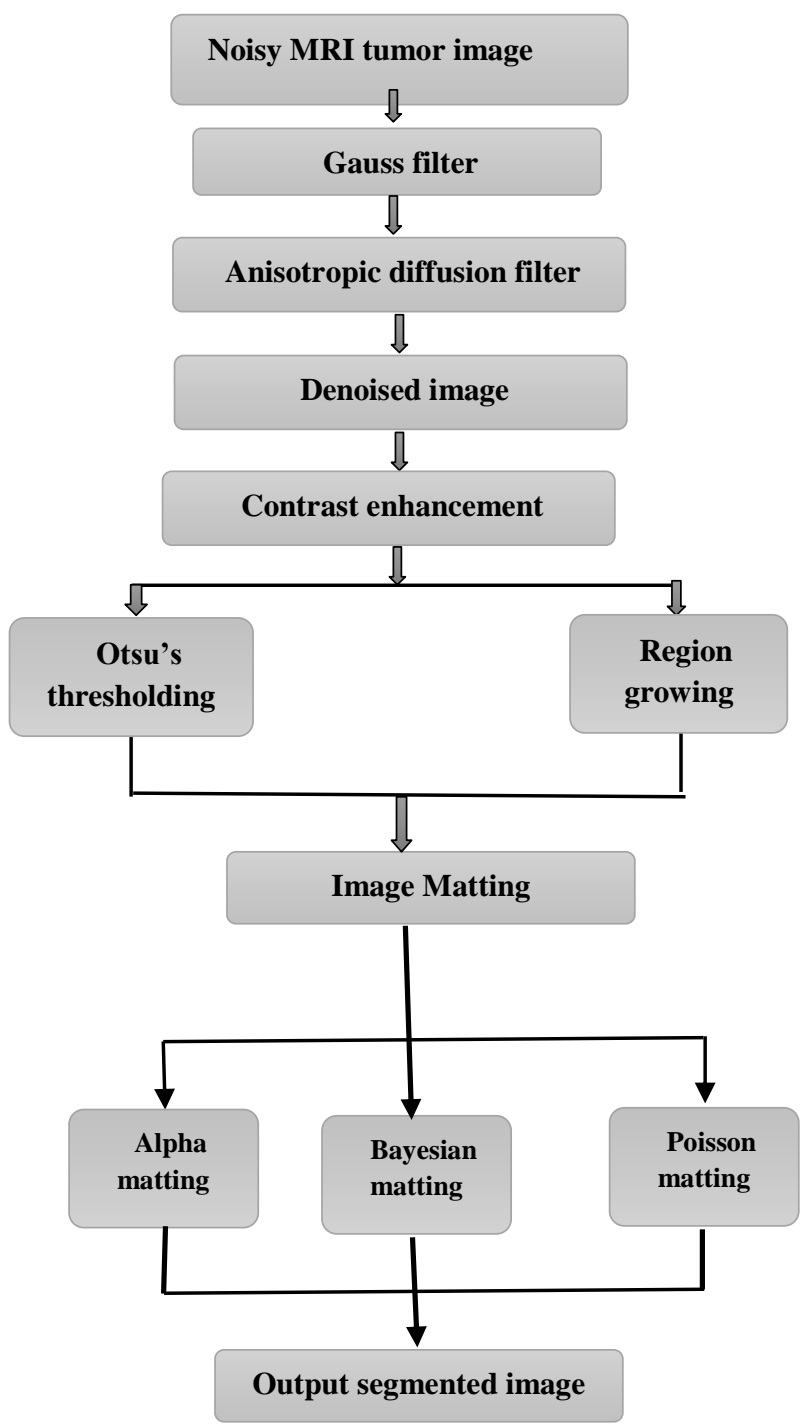

Fig 1: Flowchart of the proposed method

\subsection{Preprocessing}

Preprocessing phase of biomedical images is necessary to improve the quality of the images through noise elimination and removal of unwanted regions and making the feature extraction phase more reliable. Image denoising is a very important stage in medical imaging applications in order to enhance and recover fine details that may be hidden in the data. Removing noise from signal becomes a tough process. Hence the main objective of image denoising techniques is to remove such noise and to retain the important signal features. In particular the edges, which are the important features for MR images and thus the denoising must be balanced with edge preservation.

With reference to equation (4), the proposed method initially uses Gauss filter and later, edge preserving anisotropic diffusion filter in preprocessing stage to reduce Rician noise in tumor bearing MRI images. 


\subsubsection{Gaussian Filter}

As Rician noise can be approximated to be Gaussian with reference to the analysis made in section 2, proposed method uses Gauss filter to reduce noise. Gaussian Filter is the best option here because of its ability to remove most types of noise components. Characteristics of Gaussian method imply that it is less sensitive to extreme values and able to remove outliers without reducing sharpness of the image. Mathematically, Gaussian blur is equivalent to applying a convolution between image and Gaussian function, [17].

Smoothing is commonly undertaken using linear filters such as the Gaussian function (the kernel is based on the normal distribution curve), which tends to produce good results in reducing the influence of noise with respect to the image.

2D Gaussian distributions with standard deviation $\sigma$ is given by,

$$
G(x, y)=\frac{s}{2 \pi \sigma^{2}} \exp \left(-\frac{s^{2}+o^{2}}{2 \sigma^{2}}\right)
$$

Gaussian filter provides gentler smoothing and preserves edges better than a mean filter. The main problem with Gaussian filter is,

- Loss of fine detail

- Smoothing across boundaries

To overcome these drawbacks, anisotropic diffusion filters are used.

\subsubsection{Anisotropic Diffusion Filter}

Anisotropic diffusion filters (ADF) proposed by Perona, $\mathrm{P}$ and Malik [23], are nonlinear filters and work by using local intensity within the homogenous regions for smoothing. ADF uses a variable conductance term that regulates the diffusion at different locations in the image data [24]. The anisotropic diffusion performs a piecewise smoothing of the original signal. As a result, the propagation of information between discontinuities results in regions of constant intensity or linear variations of low frequency. Here image noise is assumed to be zero mean and Gaussian distributed. Anisotropic filter can be described by an equation (6)

$$
\frac{\partial I}{\partial t}=\operatorname{div}[C(|\nabla I|) \cdot \nabla I], \quad I(t=0)=I_{0},
$$

Where $\boldsymbol{\nabla}$ is the gradient operator, $\operatorname{div}$ ( ) is the divergence operator, $|$.$| denotes the magnitude, \boldsymbol{c}(\boldsymbol{x})$ denotes diffusion coefficient, and $\boldsymbol{I}_{\mathbf{0}}$ is the initial image.

$$
\text { where } \quad \boldsymbol{c}(\boldsymbol{x})=\frac{1}{\ln \left[\boldsymbol{e}+(\boldsymbol{x} / \boldsymbol{k})^{2}\right]}
$$

Where $\boldsymbol{k}$ denotes the edge magnitude parameter. The advantage of this filtering method is that it can smooth small discontinuities caused by background noise using gradient information and can preserve large intensity variations caused by edges.

\subsubsection{Contrast enhancement}

Contrast enhancement is a method to highlight the features of interest so that they occupy a larger portion of the displayed gray level range without distortion to other features and the overall image quality. In this paper, histogram balancing or Histogram equalization (HE) is used to improve the contrast by increasing the dynamic range of the histogram of an image. Histogram equalization assigns the intensity values of pixels in the input image such that the output image contains a uniform distribution of intensities [21]. It improves contrast by obtaining a uniform histogram. Kaur et al. [22] presented a survey of contrast enhancement techniques based on histogram equalization.

\section{REGION OF INTEREST}

ROI, usually means the meaningful and important regions in the images. The use of ROI can avoid the processing of irrelevant image points and accelerate the processing. There are lots of algorithms available for segmentation of region of interest. Researchers have found out that most information is only from some key regions in an image. If these key regions are extracted and processed, the computational speed can be highly improved. ROI stage in biomedical image analysis will improve the processing efficiency and favors easy and accurate diagnosis as irrelevant content is not taken into account.

Hence, the ROI techniques play an important role in medical images to extract only the objects of interest from an image i,e tumor regions which would enhance the speed of the analysis stage, [Bankman , 2008]. Many ROI techniques are available in literature for tumor detection. From the point of view of speed and simplicity, the proposed work uses well-known Otsu's thresholding and region growing methods for tumor ROI extraction.

\subsection{Otsu's thresholding}

Otsu's method [23] of segmentation is an optimum global thresholding method. It is optimum in the sense that it maximizes the between class variance, a well-known measure used in statistical discriminant analysis. Thresholding is a fast and computationally efficient method but it does not take into account the spatial characteristics of an image (neighborhood information). Thus thresholding is sensitive to noise and intensity in-homogeneities. In low contrast images it tends to produce scattered groups of pixels rather than connected regions and requires connectivity algorithms as a postprocessing step.

Consider a histogram normalized image with $L$ grey levels. For each grey level value $i, P(i)$ is the normalized frequency of $i$. assuming the threshold as $T$, the normalized fraction of pixels that will be classified as background and object will be,

$$
\begin{aligned}
& q_{b}(T)=\sum_{i=1}^{T} P(i) \\
& q_{0}(T)=\sum_{i=T+1}^{L} P(i)
\end{aligned}
$$

The variance of the background and object pixels will be

$$
\begin{aligned}
& \sigma_{b}^{2}(T)=\frac{\sum_{i=1}^{T}\left(i-\mu_{b}\right)^{2} P(i)}{\sum_{i=1}^{T} P(i)}=\frac{1}{q_{b}(T)} \sum_{i=1}^{T}\left(i-\mu_{b}\right)^{2} P(i) \\
& \sigma_{o}^{2}(T)=\frac{\sum_{i=T+1}^{L}\left(i-\mu_{0}\right)^{2} P(i)}{\sum_{i=T+1}^{L} P(i)}=\frac{1}{q_{0}(T)} \sum_{i=T+1}^{L}\left(i-\mu_{0}\right)^{2} P(i)
\end{aligned}
$$

Where $\mu_{b}$ and $\mu_{0}$ represent mean grey level values of back ground and object pixels respectively. The variance of the whole image is $\sigma^{2}=\sum_{i=1}^{L}(i-\mu)^{2} P(i)$

Where $\mu$ represents mean grey level value of entire image. Variance can be written as follows: 


$$
\sigma^{2}=\sigma_{w}^{2}(T)+\sigma_{B}^{2}(T)
$$

Where $\sigma_{w}^{2}(T)$ is the within class variance and $\sigma_{B}^{2}(T)$ is the between class variance. Since the total variance does not depend on $\mathrm{T}, \mathrm{T}$ minimizing $\sigma_{w}^{2}(T)$ will be the $\mathrm{T}$ maximizing $\sigma_{B}^{2}(T)$.

As Otsu's technique produces only two classes, it cannot be applied for multiple channel images. Two standard techniques such as basic global thresholding method and the standard Otsu's thresholding methods were implemented for MRI images of tumor-bearing brain in [24]. It was shown that global thresholding technique gives poor boundary of tumor ROI when trying to segment MRI images of brain tumor with one threshold value. The main drawback of global thresholding technique is the selection of the threshold value. Automatic determination of thresholds to segment complex tumor images was proposed by Chang et al. [25]. However, automatic multithresholding techniques do not provide optimal threshold values, as these are sub-optimal techniques. In such cases, region growing techniques are most preferred.

\subsection{Region Growing Technique}

In region based methods, segmentation grows from initially placed points - called seeds - by aggregating neighboring pixels or regions according to some similarity criterion. Yenwan and Higgins proposed symmetric region growing method, in which extraction of region is based on some predefined criteria and concentrates on selection of seed point [26].

The seed point can be manually selected by an operator or automatically initialized with a seed finding algorithm. Then, region growing examines all neighboring pixels and if their intensities are similar enough (satisfying a predefined uniformity or homogeneity criterion), they are added to the growing region. This procedure is repeated until no more pixels/voxels can be added to the region. Thus, it is successfully used in medical image analysis to segment different tissues, organs, or lesions and tumor regions from MR images [27].

Let $R$ be the entire region partitioned into $n$ sub regions, $R_{1}, R_{2}, \ldots, R_{n}$ such that

$$
\bigcup_{i=1}^{n} R_{i}=R
$$

$R_{i}$ is a connected region $i=1,2, \ldots, n$

$$
\begin{gathered}
R_{i} \cap R_{j}=\phi \text { for } i \neq j \\
P\left(R_{i}\right)=\text { true } \forall \\
P\left(R_{i} \cup R_{j}\right)=\text { false }
\end{gathered}
$$

For any adjacent $R_{i}$ and $R_{j}$

$P\left(R_{k}\right)$ is a logical predicate defined over the points in $R_{k}$.

Pixels or sub regions into larger regions based on predefined criteria.

\subsection{Image matting}

As the brain tumor image is surrounded by the complex tissues, it becomes very difficult to extract the tumor region. The tumor region extracted using existing methods possesses holes near the tumor boundary due to improper selection of seed point and presence of uncertainties along the tumor edges in case of thresholding process due to difficulty in finding the right threshold value. In region growing process, improper selection of seed point may produce fuzzy boundaries. To overcome this problem, image matting is used.

Matting is a process of partitioning an image into foreground and background using another region called the tri-map (unknown) or hint image which is called as matte or alpha image [28].

The matt is a grayscale layer, where values are between one and zero. The pixels present in the matte space are ambiguous. Hence it is necessary to classify each pixel in the matte into foreground or background and thereby improving the accuracy of the edges of tumor ROI. Fig 1 illustrates matting process. $\quad$ For an image matting, following equations are used.

$P=\alpha F+(1-\alpha) B$ or $B=[P-\alpha F] \div(1-\alpha)$

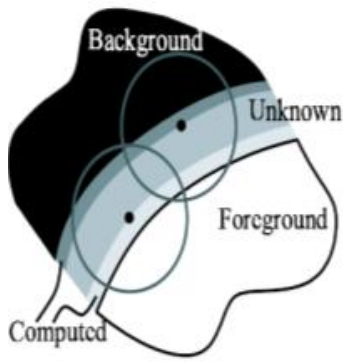

Fig 2: Diagram showing the matting process

Where, $I$ is the source image, $F$ is the foreground image, $B$ is the background image and $\alpha$ is the matte image.

Given $F \& B$ solve for $\alpha$

Use $\alpha$ to refine $F, B$

Use $F, B$ estimate to refine $\alpha$ estimate

Thus, matting is an iterative process. There are different matting techniques for image segmentation. Each one of them has its own advantages and disadvantages.

\subsubsection{Alpha Matting}

Alpha matting proposed by Ruzon et al. [29] separates the foreground and background regions in the first phase and opacity or alpha value of each pixel is tested. Later, the optimal alpha value to place the misclassified pixels in the correct region is carried out using a probability distribution interpolated between background and foreground. Ziming Zeng et al. [30] developed an unsupervised method for segmenting PET tumor images using alpha matting. In this approach, active surface modelling was used to segment the tumor region. Further, segmentation results were improved using alpha matting. The main drawback of alpha matting is high computational time complexity.

\subsubsection{Bayesian Matting}

Bayesian matting proposed by Chuang et al. [31] is based on maximum likelihood approach and finds best alpha value after testing all background and foreground sub cluster pair.In Bayesian matting technique, the matte parameters are calculated in a well-defined Bayesian framework using the maximum a posteriori (MAP) technique. MAP estimation tries to find the most likely estimates for $F, B, \propto$., Given the observation $C$, this can be expressed as a maximization over a probability distribution $P$ and then use Bayes's rule to express the result as the maximization over a sum of $\log$ likelihoods:

$$
\underset{F, \beta, \alpha}{\operatorname{argmax}} P(F, \beta, \alpha \mid C)
$$




$$
\begin{array}{r}
=\underset{F, \beta, \alpha}{\operatorname{argmax}} P(C \mid F, \beta, \alpha) P(F) P(B) \frac{P(\propto)}{P(C)} \\
=\underset{F, \beta, \alpha}{\operatorname{argmax}} L(C \mid F, \beta, \alpha)+L(F)+L(\beta)+L(\alpha)
\end{array}
$$

Where $L($.$) is the \log$ likelihood. $L()=.\log P($.$) and the$ term $P(C)$ is dropped because it is a constant with respect to the optimization parameters. The problem is now reduced to defining the log likelihoods.

$$
L(C \mid F, B, \propto), L(F), L(B) \text { and } L(\propto)
$$

The first term can be modelled by measuring the difference between the observed intensity and the intensity that would be predicted by the estimated $F, B$, and $\propto$. This log-likelihood takes care of error in the measurement of $C$. Bayesian technique has less computation time.

\subsubsection{Poisson Matting}

Poisson matting for image segmentation was proposed by Jian Sin et al. [32] uses, totally a different approach which estimates the alpha value using nearest background and foreground points. The estimated alpha value is refined by solving Poisson equation. Poisson matting directly operates on the gradient of the matte. This reduces the error caused by mis-classification of pixels in complex brain tumor images. Poisson matting consists of two steps. First, an approximate gradient field of matte is computed from the input image. Second, the matte is obtained from its gradient field by solving Poisson equations. In order to get an approximate gradient field of matte, the partial derivatives on both sides of the matting equation is considered.

$$
\nabla \mathbf{I}=(F-B) \nabla \alpha+\alpha \nabla F+(1-\alpha) \nabla B
$$

(9) It means that the matte gradient is proportional to the image gradient.

\section{EXPERIMENTAL RESULTS}

Initially, denoising of MRI tumor images corrupted by Rician noise is carried out using Gauss filter. Gauss filter reduces the noise and smoothens the image. The PSNR values for Gauss filtered images with different standard deviations $(\sigma=20,30)$ are as shown in Table.1.Further, the edge details lost in Gauss filtering process are preserved using nonlinear anisotropic diffusion filter and PSNR values are recorded. The quantitative validation is done. The second stage is the contrast enhancement which increases the visibility of fine details. This is carried out by histogram balancing because of its efficiency and simplicity.

In the third stage, the tumor region is extracted from the background using the traditional segmentation techniques such as Otsu's thresholding and region growing techniques. Segmented region and ground truth images are compared and Sensitivity analysis is done for both the techniques using entropy measures. The final stage is the image matting which incorporates three different matting techniques such as Alpha matting, Bayesian matting and Poisson matting to refine the tumor boundary information. After the matting process, sensitivity is again measured for each matting method. The readings are recorded and investigated.

\subsection{Data set}

Experiments have been conducted using brain tumor images collected from Brain-web simulated MR Datasets. In order to validate the results, the brain tumor images are corrupted with Rician noise with standard deviation of ( $\sigma=20$ and 30).
Rician noise was generated by adding Gaussian noise to real and imaginary parts and then computing the magnitude of the image.

\subsection{Validation Measure}

In this paper, the performance evaluation of different ROI techniques is carried-out using sensitivity analysis based on entropy measure. The entropy of an image can be defined as a measure of the uncertainty associated with a random variable and it quantifies, the information contained in an image. Entropy of an image $E$ returns a scalar value. According to Shannon's entropy in information theory,

$$
\text { Entropy }(E)=-\sum_{i=1}^{n} p\left(x_{i}\right) \log _{2} p\left(x_{i}\right)
$$

$p\left(x_{i}\right)$ is the probability of occurrence $i^{t h}$ pixel in a given image.

Let $E_{S}$ be the entropy of segmented image by the algorithm and $E_{g}$ be the entropy of ground truth (expert's marking). Then,

$$
\text { Error }=\left|E_{s}-E_{g}\right|
$$

Sensitivity, $S$ refers to the presence of number of true positives (number of tumor pixels) in the segmented region and is given by,

$S=1-\left[\frac{\mid \text { Error } \mid}{\text { Entropy of ground truth image }}\right]$

Higher the sensitivity, better is the efficiency of the algorithm proposed.

\subsection{Simulation Results and Observations}

The results are validated on an Intel(R) Core i5 CPU running at $2.40 \mathrm{GHz}$ with $8.00 \mathrm{~GB}$ of RAM. Software used for validation of the approach is MATLAB R2012a on MS Windows 7, 64-bit operating environment. Three images of different tumor morphology surrounded by different grey level complexities shown in Fig 3 are used for experimental purpose.

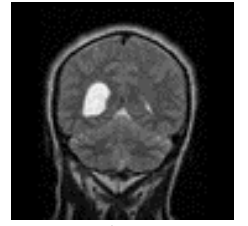

Image 1

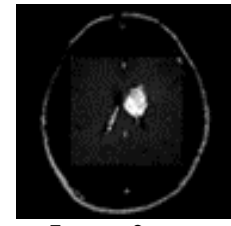

Image 2

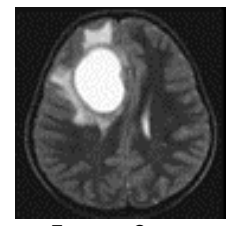

Image 3
Fig 3: MRI tumor images with different structural complexities

In Image 1, tumor region with fair contrast is surrounded by tissues with almost uniform intensity. Image 2 has a tumor with dark background whereas Image 3 has a bright tumor region surrounded by tissues of different intensities. In this paper, implementation is carried out for all the three images shown in Fig 3. Experimental results for the performance measures: PSNR and sensitivity are given in Table 1 and Table 2 respectively. However, the processed images for noise removal and ROI extraction are shown only for Image 1 . The results of the denoising stage are shown in Table 1 for the T1 weighted images corrupted by Rician noise with standard deviation (SD), $\sigma=20$ and 30. The experimental results have 
demonstrated the satisfactory performance for noise removal using Gauss filter followed by ADF.

Table 1: Experimental results of denoising

\begin{tabular}{|c|c|c|c|c|c|}
\hline $\begin{array}{c}\text { Performance } \\
\text { metric }\end{array}$ & $\begin{array}{c}\text { SD } \\
\sigma\end{array}$ & $\begin{array}{c}\text { Tumor } \\
\text { Images }\end{array}$ & $\begin{array}{c}\text { Noisy } \\
\text { Images }\end{array}$ & $\begin{array}{c}\text { Gauss } \\
\text { filter }\end{array}$ & ADF \\
\hline \multirow{3}{*}{ PSNR (DB) } & \multirow{3}{*}{20} & Image 1 & 18.35 & 23.3 & 24.1 \\
\cline { 3 - 6 } & & Image 2 & 19.8 & 24.8 & 25.3 \\
\cline { 2 - 6 } & \multirow{2}{*}{30} & Image 3 & 17.67 & 23.9 & 24.6 \\
\cline { 2 - 6 } & Image 1 & 16.8 & 22.5 & 23.2 \\
\cline { 3 - 6 } & & Image 2 & 15.6 & 23.7 & 23.9 \\
\cline { 2 - 6 } & Image 3 & 16.3 & 23.3 & 24.1 \\
\hline
\end{tabular}

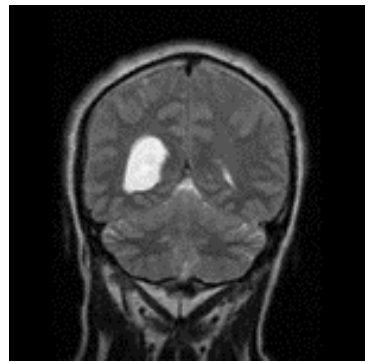

(a)

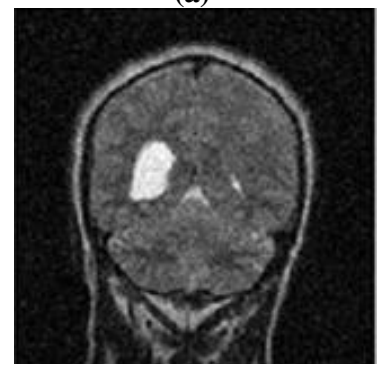

(c)

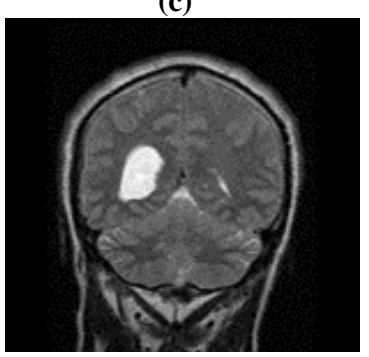

(e)

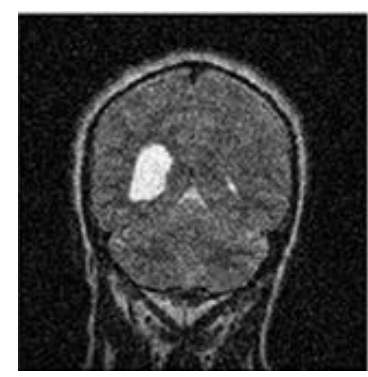

(b)

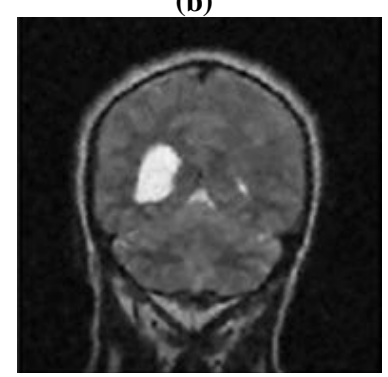

(d)
Fig 4: Experimental results of image denoising: (a) Original image, (b) Noisy image (c) Gauss filtered imag

\section{(d) ADF filtered image (e) Contrast enhanced image}

Further, the contrast level of the filtered image is considerably improved using histogram equalization as shown in Fig 4 (e).

The main aim of this work is to investigate the suitability of ROI methods in tumor extraction. This work reports an empirical comparison of three different image matting techniques in context of refining the tumor boundary. The denoised-enhanced image shown in Fig (5a) is segmented using Otsu's thresholding and region growing techniques.

Segmented outputs are shown in Fig. (5c) \& (5d) and sensitivity is calculated using the entropy of the segmented output \& the entropy of expert's ground truth image shown in Fig. (5b), using equation (10). It can be observed that in Otsu's thresholding, $\mathrm{S}$ value is different for each image and image with more details (Fig 3, Image 3) has less S value compared to other two images. Whereas region growing method has better $\mathrm{S}$ value for Image 3 but lesser sensitivity for (Image 1 and Image 2). Thus Otsu's method may produce better results for good contrast tumor images and region growing method gives satisfactory results for more complex tumor regions. In either approaches poor boundary exists based on the image grey level complexity. It is therefore necessary to refine the tumor boundary for improving the accuracy of tumor extraction. In this paper, performance of three image matting techniques have been investigated and thereby to choose the best matting technique to reduce boundary ambiguities. In any matting technique, a Trimap or unknown region (band of ambiguous pixels) or $\alpha$ has to be generated to place the misclassified pixels into proper foreground and background. Fig. (5f) \& Fig. (5j) show the Trimap images for Otsu's and region growing methods.

In Alpha matting, $\alpha$ is estimated using the pixel values of foreground $(\mathrm{F})$ and background (B). Further, unknown $(\alpha)$ pixels are assigned to $\mathrm{F}$ or $\mathrm{B}$ based on pixel position and pixel value. The process repeats till all the pixels are assigned to the respective regions. Alpha matting process is iterative and consumes large amount of time as specified in Table 2 for 500 iterations. Bayesian matting uses Bayes' rule to estimate $\alpha$. It can be observed in Table 2, this technique results in good sensitivity compared alpha matting with less processing time (600s). Whereas Poisson matting estimates $\alpha$ based on Poisson rule and gives the best results in combination with region growing method shown in Fig. (4m) with little more execution time (720s) compared to Bayesian matting. It can also be noted from Table 2 that noisy images result in poor sensitivity and hamper the analysis stage. Hence denoising is a necessary stage in medical image analysis.

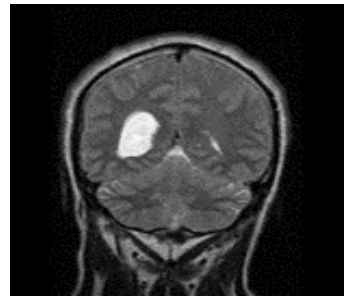

(a)

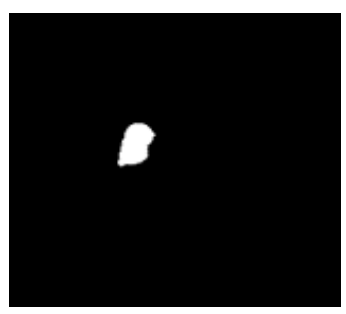

(c)

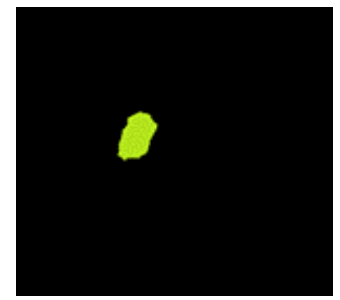

(b)

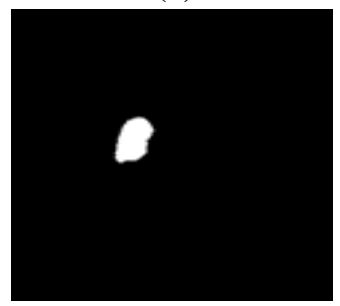

(d) 


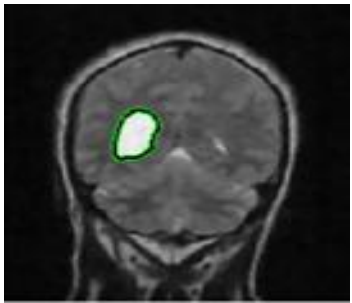

(e)

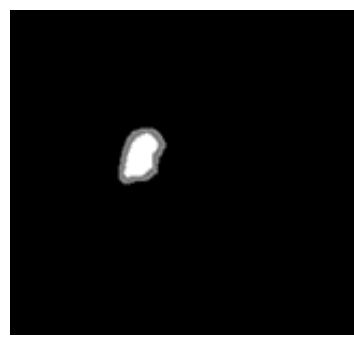

(j)

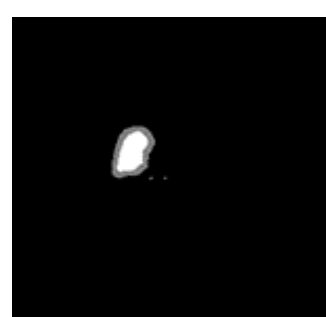

(f)

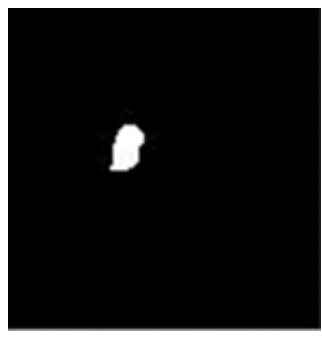

(k)

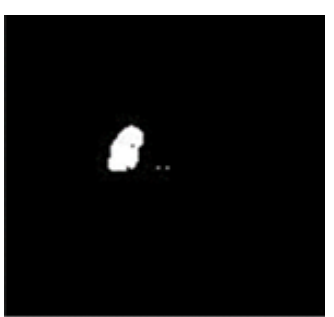

(g)

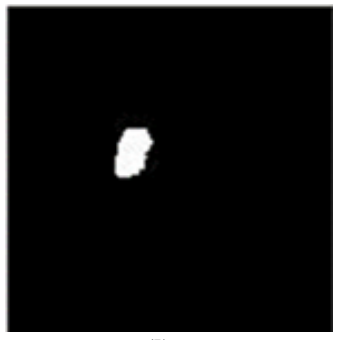

(l)

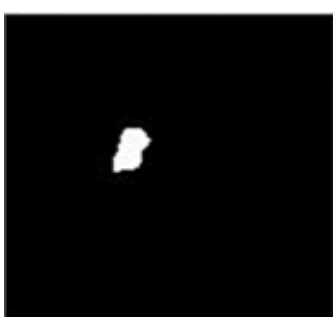

(h)

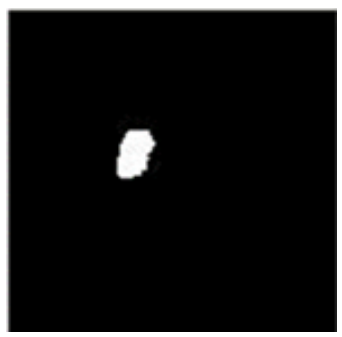

(m)

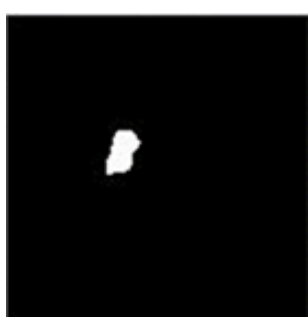

(i)

Fig 5: (a) Enhanced image, (b) Ground truth, (c) Otsu's thresholding, (d) Region growing, (e) ROI (f) Trimap for Otsu's (g) Alpha Otsu's, (h) Bayesian Otsus, (i) Poisson Otsus (j) Trimap for region growing (k) Alpha Region growing (l) Bayesian Region growing (m) Poisson Region growing

Table 2: Experimental results of different image matting techniques

\begin{tabular}{|c|c|c|c|c|c|c|c|c|c|}
\hline \multirow{2}{*}{$\begin{array}{c}\text { Performance } \\
\text { metric }\end{array}$} & \multirow[t]{2}{*}{ Tumor Images } & \multirow{2}{*}{$\begin{array}{l}\text { Otsu } \\
\text { 's } \\
\text { meth } \\
\text { od }\end{array}$} & \multirow{2}{*}{$\begin{array}{l}\text { Region } \\
\text { growing } \\
\text { method }\end{array}$} & \multicolumn{3}{|c|}{ Otsu's with matting } & \multicolumn{3}{|c|}{ Region growing with matting } \\
\hline & & & & $\begin{array}{c}\text { Alpha } \\
\text { matting }\end{array}$ & $\begin{array}{c}\text { Bayesian } \\
\text { matting }\end{array}$ & $\begin{array}{l}\text { Poisson } \\
\text { matting }\end{array}$ & $\begin{array}{c}\text { Alpha } \\
\text { matting }\end{array}$ & $\begin{array}{c}\text { Bayesian } \\
\text { matting }\end{array}$ & $\begin{array}{l}\text { Poisson } \\
\text { matting }\end{array}$ \\
\hline \multirow{4}{*}{$\begin{array}{c}\text { Sensitivity } \\
(\%)\end{array}$} & Image 1 & 91.2 & 91 & 90.4 & 93 & 92 & 92 & 96 & 97 \\
\hline & Image 2 & 91.6 & 91 & 92 & 94 & 94 & 96 & 98 & 98 \\
\hline & Image 3 & 90.6 & 92 & 93.7 & 92.6 & 95 & 93 & 95 & 96 \\
\hline & Noisy Image 1 & $\begin{array}{c}88.9 \\
2\end{array}$ & 87.76 & 87.89 & 88.87 & 87.67 & 87.6 & 88.3 & 87.2 \\
\hline $\begin{array}{c}\text { Execution } \\
\text { time(s) } \\
500 \text { iterations }\end{array}$ & $\begin{array}{l}\text { Image size } \\
256 \times 256\end{array}$ & & & $1200 \mathrm{~s}$ & $600 \mathrm{~s}$ & $700 \mathrm{~s}$ & $1100 \mathrm{~s}$ & $650 \mathrm{~s}$ & $720 \mathrm{~s}$ \\
\hline
\end{tabular}

\section{CONCLUSIONS}

This paper investigates the performance evaluation of three different image matting techniques employed to refine poor tumor boundaries in complex MRI brain tumor images corrupted by Rician noise. Denosing results show that image quality improves with a combination of Gauss filter and ADF filter. It can be seen that traditional segmentation techniques alone would not fetch satisfactory results. Further accuracy of tumor boundary is found to be marginally improved using matting techniques. Extraction results reveal that Bayesian matting and Poisson matting techniques combined with region growing would provide satisfactory results in less time compared to alpha matting. Further, a combination of Poisson matting and region growing techniques yield better sensitivity compared to Bayesian matting. Hence for high complex images, Poisson matting with region growing refines the tumor boundary compared to other matting techniques. Future work can be extended to investigate the performance of matting techniques on 3D color images of MRI and other imaging modalities such as: CT and Ultrasound.

\section{REFERENCES}

[1] El-Sayed A. El-Dahshan "Computer-aided diagnosis of human brain tumor through MRI": A survey and a new algorithm", Expert Systems with Applications, Elsevier, Volume 41, Issue 11, pp. 5526-5545., September 2014.

[2] Halder, A., Giri, C., Halder, A. Brain Tumor Detection Using Segmentation Based Object Labeling Algorithm. Electronics Communication and Instrumentation (ICECI) 201. International Conference, vol. no., pp.1, 4. 16-17 Jan. 2014.

[3] Jason J., Corso., Eitan Sharon., ShishirDube., Suzie ElSaden., Usha Sinha., Alan Yuille. Efficient Multilevel Brain Tumor Segmentation with Integrated Bayesian Model Classification. IEEE Transactions on Medical imaging Vol., 27, Issue 5. pp. 629-640. May 2008.

[4] Tao Wang., Irene Cheng., AnupBasu. Fluid Vector Flow and Applications in Brain Tumor Segmentation. IEEE Transactions on Biomedical Engineering, Vol., 56, No. 3, March 2009.

[5] Tanoori, B., Azimifar, Z., Shakibafar, A., and Katebi, S. Brain Volumetry: An active contour model-based segmentation followed by SVM-based classification. 
Computers in Biology and Medicine, Vol., 41(8), pp. 619-632, 2011.

[6] Jafari, M., and Kasaei, S. Automatic Brain Tissue Detection in MRI Images Using Seeded Region Growing Segmentation and Neural Network Classification. Australian Journal of Basic and Applied Sciences. Vol., 5(8), pp. 1066-1079, 2011.

[7] BaidyaNathSaha. Nilanjan Ray., Russell Greiner., Albert Murtha., Hong Zhang. Quick Detection of Brain Tumors and Edemas: A Bounding Box Method Using Symmetry. Computerized Medical Imaging and Graphics. ISSN 08956111, pp. 95-107, 2012.

[8] ShiyongJi. Benzheng Wei., Zhen Yu., Gongping Yang and Yilong Yin. A New Multistage Medical Segmentation Method Based on Super pixel and Fuzzy Clustering. Hindawi Publishing Corporation Computational and Mathematical Methods in Medicine. Article ID 747549. 2014.

[9] Meiyan Huang., Wei Ynag., YaoWu,JunJiang., Wufan Chen. Brain Tumor Segmentation Based on Local Independent Projection-Base Classification. IEEE transactions on Biomedical Engineering, Vol., 61, No. 10, pp. 2633-2645, October 2014.

[10] Macovski. A. Magnetic Resonance in Medicine. 36 (3). 494. doi:10.1002/mrm.1910360327, 1996.

[11] Nowak. R, Wavelet based Rician noise removal in MRI, IEEE Transactions on Image Processing 8 (10). 1408. 1999.

[12] Nobi. M.N. and Yousuf. M. A. A New Method to Remove Noise in Magnetic Resonance and Ultrasound Images. Journal of Scientific Research. www.banglajol.info/index.php/JSR, 3 (1). 81-89., 2011.

[13] Chan., R. H., Ho., C., and Nikola. M. Salt and pepper noise removal in CT images using median filtering, IEEE Transactions on image processing, 14 (10), 1479. 2005.

[14] Milindkumar V., Strode. Dr. Prashant R., Deshmukh. Performance Evaluation of Rician Noise Reduction Algorithm in Magnetic Resonance Images. Journal of Emerging Trends in Computing and Information Sciences. Vol., 2 CIS Journal ISSN 2079- 8407. pp. 3944., Special Issue 2010-11.

[15] IsshaaAarya., Danchi Jiang., and Timothy Gale. Signal Dependent Rician Noise Denoising Using Nonlinear Filter. Lecture Notes on Software Engineering, Vol., 1, No., 4, November 2013

[16] Arsenault H. and Denis, M. Image processing in SignalDependent noise. Can. J. Phys., Vol., 61, No., 2, pp. 309-317., 1983.

[17] Gedraite, E.S., Hadad, M. Investigation on the Effect of A Gaussian Blur In Image Filtering and Segmentation. IEEE conference publication held at Zadar. ISSN- 13342630. Issue Date. 14-16. September 2011.

[18] Perona, P., and Malik, J. Scale-space and edge detection using anisotropic diffusion. IEEE Trans. Pattern. Anal. Machine. Intel. Vol., 12. No., 7. pp. 629- 639. July 1990.
[19] Gerig, O., Kubler, R., Kikinis, and Jolesz F.A. Nonlinear anisotropic filtering of MRI data. Medical Imaging. IEEE Transactions on, 11(2):221-232, 1992.

[20] Krissian. And S. Aja-Fernndez, Noise-Driven Anisotropic Diffusion Filtering of MRI. IEEE Transactions on Image Processing, 18(10):2265-2274, 2009.

[21] Garg, R., Mittal, B., Garg, S. Histogram Equalization techniques for image enhancement. IJECT. Vol., 2, pp. 107-111., 2011.

[22] Kaur, M., Kaur, J., Kaur, J. Survey of Contrast Enhancement Techniques Based On Histogram Equalization. International Journal of Advanced Computer Science and Applications, Vol. 2, 2011.

[23] Otsu. N. A Threshold Selection Method from Gray-level Histograms. IEEE Transactions on Systems, Man \& Cybernatics, Vol., 9, Issue 1. pp. 62-66., 1979.

[24] Tirpude, NN*. Welekar RR. Effect of Global Thresholding on Tumor-Bearing Brain MRI Images. International Journal of Engineering and Computer Science ISSN: 2319- 7242. Vol., 2. Issue 3. pp. 728-731. March 2013.

[25] Yen, C., Chang, F.J., Chang, S. A New Criterion for Automatic Multilevel Thresholding. IEEE Transactions on Image Processing. I, P-4., pp. 370-378., 1995.

[26] Gonzalez, R., Woods, R., and Eddins, S. Digital Image Processing Using MATLAB. Pearson Education.

[27] Weglinski, T., and Fabijanska, A. Brain Tumor Segmentation from MRI Data Sets Using Region Growing Approach. In Proceedings of the 7th International Conference on Perspective Technologies and Methods in MEMS Design (MEMSTECH '11). pp. 185-188. May 2011

[28] Levin, A., Lischinski, D., and Weiss. Y. A Closed Form Solution to Natural Image Matting. Hebrew University Technical Report. 2006.

[29] Ruzon, M., Tomasi C. Alpha Estimation in Natural Images. In Proceedings of the IEEE Conference on Computer Vision and Pattern Recognition. Vol., 1, pp. 18.25 , June 2000

[30] Ziming Zeng, JueWang, C., BernieTiddeman, A., and ReyerZwiggelaar. Unsupervised Tumour segmentation in PET using local and global intensity-fitting active surface and alpha matting. Computers in Biology and Medicine. Elsvier, 2013.

[31] Chuang, Y.Y., Brain, C., Salesin, D. H., Szelsiki, R. A Bayesian Approach to Digital Matting. In IEEE Computer Society Conference on Computer Vision and Pattern Recognition, CVPR. Vol., 2, pp. 264-71. 9th-14th December 2001.

[32] Jian Sin, JiyayaJiya, Chi- Keung Tang, Heung-Yueng Shum. Poisson Matting, SIGGRAPH 2004. ACM Transaction on Graphics. 23. 3., pp. 315-321., April 2004. 